\title{
CONVERSION : NON-RÉCIPROQUE - RÉCIPROQUE. VARIANTES SYNTAXIQUES
}

\begin{abstract}
Nkollo Mikołaj, Conversion: non-réciproque - réciproque. Variantes syntaxiques [Reciprocal transformation. Syntactic varieties], Studia Romanica Posnaniensia, Adam Mickiewicz University Press, Poznań, vol. XXXVII/2: 2010, pp. 15-27. ISBN 978-83-232-2189-0. ISSN 0137-2475. DOI 10.2478/ v10123-010-0011-5.

The present paper is devoted to the concept of reciprocity and to reciprocal expressions, which are divided into two main classes. Those belonging to the first of them signify reciprocity on their own, i.e. without any additional reciprocal markers. The other class contains expressions that acquire ability to refer to reciprocal states of affairs only as a result of transformation. Different reciprocal markers - grammatical, lexical and quantifying - are singled out and thoroughly characterized. Their use results in converting nonreciprocal signs into reciprocal ones. They are applied according to the part of speech of a modified term. The conclusion to be drawn from this analysis revolves around the discrepancy between morphological and syntactic means of reciprocal transformation. While the former ones are rather scanty, the latter are largely used in contemporary French.
\end{abstract}

\section{LA RÉCIPROCITÉ ET LES CONSTRUCTIONS RÉCIPROQUES - CARACTÉRISTIQUE SÉMANTIQUE}

Qualifiée de propriété structurale de certains syntagmes verbaux, la réciprocité n'est pas moins une propriété sémantique de plusieurs expressions des langues humaines. Elle peut, dans des conditions syntaxiques particulières, s'étendre aux combinaisons de ces expressions avec leurs partenaires syntagmatiques. Occasionnellement, elle est susceptible de caractériser aussi les parties de ces expressions. C'est donc surtout au niveau sémantique que sera localisée la réciprocité dans le présent article. Plus spécifiquement, seront considérées comme réciproques les signes qui nomment des états de choses engageant au moins deux participants différents, dont chacun se trouve dans une même situation à l'égard de l'autre. L'étiquette «participant » sert à nommer des personnes, des objets, des qualités ou des états de choses mis en correspondance et ne préjuge en rien leur statut ontologique. Il doit y avoir entre les participants $-x$ et $y$ - à un tel état de choses (ou relation $R$ ) une dépendance telle que l'existence de 
$R$ entre $x$ et $y$ signifie nécessairement que cette relation est valable également dans le sens opposé, c.-à.-d. entre $y$ et $x$ (Pogonowski, 1993, p. 7). C'est ainsi que les noms analogie, opposition, équivalence, du fait qu'ils établissent une certaine parité entre les participants aux états de choses qu'ils signifient, seront comptés au nombre d'expressions réciproques. La dépendance mutuelle de deux objets qui sont analogues, qui s'opposent entre eux ou qui s'équivalent distingue sémantiquement ces noms de ceux qui signifient une relation uni-directionnelle, orientée d'un objet à l'autre, mais non à l'inverse.

L'identité des positions qu'occupe chaque participant envers l'autre signifie que les deux font la même choses ou possèdent, l'un au même titre que l'autre, une seule propriété ou qu'il se passe avec eux une même chose. Cette égalité de leurs statuts ne suffit pourtant pas à garantir la réciprocité. Plusieurs signes, dès l'instant où ils sont accompagnés des éléments nominaux collectifs ou au pluriel (Ces deux nouveaux modèles étaient particulièrement regardés par les clients / par la clientèle), fondent des phrases qui renvoient à plusieurs activités d'un même type concernant plusieurs individus. Il n'est cependant pas légitime d'en conclure au caractère réciproque de ces situations. En effet, le fait de regarder attentivement une voiture n'établit aucune interdépendance entre les individus formant le public. Même s'ils forment un groupe cohérent, chacun d'entre eux peut participer à la situation à sa guise, indépendamment de ce que font d'autres membres du groupe.

Parmi les noms de situations réciproques, un ensemble d'expressions, appelées reciproca tantum par la suite, se singularise par ses propriétés linguistiques. Conformes aux critères mentionnés ci-dessus, les signes formant cette classe ont des significations associables à des situations nécessairement réciproques. De ce fait, ils ne doivent pas être accompagnés des exposants additionnels de cette valeur sémantique. Il n'y a rien d'extraordinaire dans cette découverte. Les situations nommées égalité, proximité, identité, amitié, conversation, etc. instaurent entre leurs participants une interdépendance. Il s'agit des situations réciproques et, en même temps, reversibles. Ces noms évoquent des concepts fondamentaux qui trouvent des appellations dans la presque totalité des langues humaines examinées jusqu'à maintenant. Certains signes correspondant aux concepts mentionnés ici font partie du fameux répertoire de «primitifs sémantiques » de Wierzbicka (1993, p. 17). Ceci permet de supposer qu'ils deviennent facilement le point de départ de plusieurs instances de dérivation sémantique. Il peut s'agir p.ex. de la subordination du concept d'égalité à celui de cause ; c'est ainsi qu'apparaît le signifié du verbe du type égaliser. Il doit être noté que les correspondants négatifs de ces expressions nomment aussi des situations réciproques. Ces propriétés sémantiques des reciproca tantum sont indépendantes du partage en parties du discours. Elles caractérisent donc au même degré les noms, les adjectifs et les verbes. Dans des conditions syntaxiques et lexicales particulières, ces caractères deviennent propres même à des expressions non-autonomes - les prépositions et les conjonctions. C'est ainsi que les deux objets mis en correspondance par la relation 
d'égalité sont par définition mutuellement dépendants. Quelque soit le mode d'expression de leur statut (Il y a égalité entre Jean et Pierre ; Pierre et Jean sont égaux, Jean et Pierre s'égalent), la relation de l'un envers l'autre vaut, en vertu de la nature même du concept d'égalité, aussi dans le sens opposé.

L'originalité des signes représentant cet ensemble consiste dans leur aptitude à nommer une seule situation extra-linguistique, non divisible en parties du même type que la situation globale. Il ne sera pas inutile d'illustrer cette propriété avec un exemple. Si deux équipes s'affrontent dans un match de football qui finit sur le score 0 à 0 , le résultat obtenu à l'issue de la partie par l'équipe A contre l'équipe $\mathrm{B}$ ne se laisse pas dissocier du résultat de B contre A. Il est question toujours d'un seul et même événement. Autrement dit, il n'y a pas de matchs ou de matchs nuls « unilatéraux ». Il s'agit d'une seule situation extra-linguistique, orientée simultanément dans deux directions. Il est impossible d'en extraire deux parties dont chacune représente la situation de l'une des équipes, mais non la situation de l'autre. La signification des reciproca tantum, à l'opposé de celle des autres relations dyadiques, est donc indifférente quant à la façon dont sont ordonnés les éléments formant une paire. Alors que le sens des expressions non-réciproques (concession, victoire, avantage, défaite, etc.) demande qu'il existe une hiérarchie unidirectionnelle entre les participants, cette exigence disparaît dès qu'on a affaire aux noms de relations réciproques. La modification de l'ordre des éléments formant l'ensemble signifié par une expression non-réciproque équivaut à une autre relation (Michel a insulté son professeur d'anglais en actes et en paroles représente une autre situation extra-linguistique que Le professeur d'anglais a insulté Michel en actes et en paroles). Cet agencement perd son importance dès lors que la réciprocité, qui est reversible, entre en jeu. Cette caractéristique est complétée par une dernière contrainte - les participants à une situation réciproque doivent être comparabales entre eux. Leur proximité ontologique doit être telle que soit acceptable la compression de leurs noms à un seul terme au pluriel. En témoignent les exemples:

Le président est sur ce point en désaccord avec le tribunal.

= Le tribunal est sur ce point en désaccord avec le président.

= Ils sont en désaccord sur ce point (ils = le président et le tribunal).

Le président est sur ce point en désaccord avec les dix commandements.

??? Les dix commandements sont sur ce point en désaccord avec le président.

??? Ils sont en désaccord sur ce point (ils = le président et les dix commandements).

Les propriétés décrites ci-dessus ont une solide confirmation emprique et se laissent observer au niveau syntaxique. Grâce au caractère non ordonné des ensembles auxquels s'appliquent les reciproca tantum les éléments nominaux qui accompagnent cette classe de signes peuvent changer librement de place. Il n'en résulte aucun changement sémantique. En même temps, la forme du nom de la situation réciproque reste intacte. Ce trait permet d'opposer les reciproca tantum aux relations qui exigent que soient ordonnés les ensembles auxquels elles s'appliquent. La permutation des noms des participants exige dans ce cas-là une transformation passive ou bien l'emploi des 
noms de relations converses. La forme que revêt l'élément prédicatif se voit donc toujours modifiée. La permutation des éléments nominaux qui n’entraîne aucun changement référentiel est attestée par les exemples polonais ci-dessous.

Golota stoczy walkę z Riddickiem Bow w Las Vegas.

Riddick Bow stoczy walke z Gotota w Las Vegas.

Jacek i Krystyna majq się wyraźnie ku sobie.

Krystyna i Jacek maja się wyraźnie ku sobie.

Pańskie zeznanie jest analogiczne do tego, co powiedziała nam pańska żona.

To, co powiedziała nam pańska żona, jest analogiczne do pańskiego zeznania.

L'autre caractéristique des constructions réciproques - la possiblité d'insérer les noms des participants à l'intérieur d'un seul constituant (Jacques et Sylvie se querellent à tout bout de champ) - sera aussi jugée comme la manifestation au niveau syntaxique de leur égalité. La coordination, c.-à.-d. la structure où aucun terme ne détermine l'autre ni n'en n'est déterminé (en l'occurrence Jacques n'est nullement subordonné à Sylvie et vice-versa), implique la parité de ces termes. Une telle organisation structurale reflète l'identité des situations de chaque participant à l'égard de l'autre. La compression, régulièrement pratiquée dans des constructions réciproques, des noms de participants à un terme unique (Ils se querellent à tout bout de champ où $I l s=$ Jacques et Sylvie) sera interprétée de la même façon.

Les reciproca tantum ne sont pourtant pas l'unique classe de noms de relations réciproques. D'autres noms et constructions aux propriétés sémantiques analogues sont introduites dans la langue par la voie transformationnelle. Initialement dépourvus de la capacité de nommer des situations réciproques, ils proviennent de la modification des expressions inscrites déjà dans le dictionnaire. Ces modifications permettent de passer p.ex. de la forme saluer à se saluer, de peine à s'infliger une peine, et puis à s'infliger mutuellement des peines; de injuste envers quelqu'un à injustes l'un envers l'autre, etc. Elles seront toutes, conformément à ce qui a été annoncé dans le titre de cet article, logées sous l'étiquette commune de «conversion : non-réciproque - réciproque ». Plus spécifiquement, les transformations en question sont le produit de la conjonction de deux relations identiques dont au départ aucune n'est réciproque. La jonction de ces relations réussit parce qu'elles sont de même nature. Les objets qui y participent sont chaque fois différemment ordonnés à l'intérieur des ensembles auxquels chacune des relations s'applique. S'il y a amour mutuel entre Pierre et Marie, on a affaire à un état de choses complexe, composé de deux parties : amour « unilatéral » de Pierre envers Marie et d'un autre amour « unilatéral » qui concerne la paire ordonnée d'éléments $<$ Marie, Pierre $>$. Il s'agit, dans chacun des ensembles, des mêmes participants auxquels échouent des rôles différents. Sont donc mises ensemble deux relations uni-directionnelles.

Ceci permet de définir l'opposition fondamentale entre cette classe des noms réciproques et les reciproca tantum. Tandis que les seconds renvoient à une situation indivisible, les expressions converties font référence à au moins deux situations de même 
nature. Leurs référents ont donc une structure bipartie. Chacune de leurs composantes correspond à une des directions dans lesquelles sont orientées les deux situations soumises à la jonction. Les opérations qui donnent naissance aux noms dérivés de situations réciproques sont parallèles aux modifications affixales réalisées en français grâce à l'emploi de co-, entre- et inter-. P.ex. le nom coexistence renvoie en réalité aux deux existences qui ne dépendent pas nécessairement l'une de l'autre. Chacun d'entre les individus mis en correspondance peut avoir une existence autonome. Le préfixe co- introduit ici des valeurs de simultanéité et de réciprocité ou collectivité (Zaliznjak et Shmelev, 2007, pp. 212-216).

Le caractère biparti des référents des signes obtenus à l'issue de la conversion est confirmée par la synonymie des phrases fondées sur cette classe d'expressions réciproques et des constructions polyphrastiques. Ces dernières sont des phrases complexes, formées par la coordination des propositions dont chacune représente une des situations soumises à la jonction. La même propriété y est attribuée d'abord à l'un, puis à l'autre participant. Vu l'identité de ce que font et de ce qui advient aux participants à cette sorte de situations complexes, la deuxième partie des constructions polyphrastiques abonde ordinairement en pronoms anaphoriques, omissions délibérées ou, au contraire, éléments récurrents. Il existe dans la langue des signes dont la vocation est justement de figurer dans ce type de constructions, p.ex. vice-versa, à l'inverse. En témoignent les exemples polonais

Maria kocha Janka, a Janek ja.

= Maria i Janek się kochaja.

Życzyliśmy Nowackiemu udanego urlopu, a on zrewanżowat się tym samym.

= Życzyliśmy sobie z Nowackim udanego urlopu.

Darek nie znosi naszego kota, a nasz kot nie znosi go.

= Darek i nasz kot nie znosza się.

Piotr byt uprzedzony wobec swoich kolegów i vice versa.

= Piotr i jego koledzy byli wobec siebie wzajemnie uprzedzeni.

Les constructions polyphrastiques seront considérées comme sémantiquement primaires. Cette affirmation s'appuie justement sur l'observation des propriétés sémantiques des expressions réciproques dérivées. En effet, leur emploi finit par réduire à une seule phrase deux propositions, chacune représentant à l'origine une des relations mises ensemble. À l'opposé des constructions polyphrastiques, jugées plus iconiques, les noms de relations réciproques formés transformationnellement réalisent le principe d'économie qui régit certains aspects de la communication linguistique. L'emploi des constructions réduites semble surtout plus commode, particulièrement là où le nombre des participants à la situation dépasse deux. L'expression de ces situations au moyen de stratégies polyphrastiques serait vouée à des réitérations fastidieuses, ce qui contrevient aux principes de cohérence discursive (Les enfants, les adolescents et les vieillards se tenaient par les mains représenterait donc la conjoction de Les enfants tenaient les mains des adolescents et des vieillards, les adolescents 
tenaient les mains des enfants et des vieillards et les vieillards tenaient les mains des enfants et des adolescents).

Au niveau distributionnel, la différence la plus nette entre les deux classes d'expressions réciproques concerne leur combinabilité avec les déterminants numériques, surtout les numéraux cardinaux et les indications multiplicatives (contenant la forme fois). Une fois cette combinaison effectuée, les effets obtenus avec l'un et l'autre type d'expressions ne sont pas les mêmes. Les phrases Ils se saluèrent deux fois ou Ils saluèrent deux fois l'un l'autre, bâties sur une expression réciproque dérivée sont interprétables de deux façons. Soit chaque participant a salué l'autre une fois (il y a en somme deux saluts « unilatéraux »), soit chacun l'a fait deux fois (il y a alors quatre saluts). Par contre, l'indication du nombre ou de la multiplicité en compagnie des reciproca tantum rétrécit le nombre des interprétations accessibles. Ils se rencontrèrent deux fois exclut la situation composée des quatre rencontres. Comme il n'existe pas de rencontres « unilatérales » (qui concernent un individu, mais excluent la participation de l'autre), l'unique possibilité consiste dans deux rencontres « bilatérales ». Ceci n'est pas étonnant si l'on se souvient du caractère de la prédication réalisée par l'une et l'autre classe de signes. En outre, les reciproca tantum sont suceptibles de faire partie d'une gamme plus large de modèles structuraux. Éléments centraux des constructions simples (avec l'insertion des noms des participants à l'intérieur d'un seul constituant), ils sont aussi capables de fonder des constructions discontinues (où les noms des participants appartiennent à deux syntagmes différents, hiérarchiquement non égaux Pierre est le rival de Jacques, Pierre a rencontré Jacques). Par contre, les expressions réciproques dérivées figurent à peu près uniquement dans des constructions simples.

\section{LES MÉCANISMES DE CONVERSION}

La compression de deux relations d'un type, mais différamment ordonnées (Pierre respecte Jean et Jean respecte Pierre), à une seule forme nommant les deux directions (Pierre et Jean se respectent) s'accompagne obligatoirement de l'emploi de certains signes. Leur rôle consiste à conférer aux noms des relations mises ensemble un caractère réciproque. Pourtant, ces indicateurs de réciprocité ne suffisent pas, eux-mêmes, à nommer une situation extra-linguistique. Ils n'acquièrent cette aptitude qu'au moment où ils sont combinés aux noms de relations non-réciproques. Il se crée de la sorte des configurations d'éléments qui signifient, les uns conjointement avec les autres, un fragment de la réalité. Chacun d'entre ces éléments y contribue à sa guise. Ce mode de signifier sera appelé « co-signification ». La conversion : non-réciproque - réciproque s'appuie sur l'emploi des moyens lexicaux, quantificatifs ou bien grammaticaux. Ce partage fait appel au degré d'autonomie articulatoire, syntaxique et sémantique des indicateurs de réciprocité. Les éléments lexicaux sont capables de jouer le rôle du noyau d'un syntagme ou bien forment, eux-mêmes, des syntagmes. Certains d'entre 
eux peuvent, dans des conditions discursives spéciales, prendre la relève de toute une phrase (- Je vous souhaite un heureux événement pour bientôt - Et réciproquement; Le député britannique adressait un blâme à son homologue français et vice-versa). Par contre, les moyens dits " grammaticaux » ne connaissent pas d'existence autonome. Ils s'ajoutent obligatoirement à d'autres termes formant une construction réciproque. Il est possible d'en distinguer plusieurs types. Les éléments affixaux, les moins autonomes, font toujours partie d'autres mots ou parties de mots. Parmi les moyens non-affixaux, on délimite les éléments clitiques et toniques. Les premiers ont une mobilité plus grande que les affixes, mais doivent toujours apparaître en compagnie d'autres termes. Au plan articulatoire, ils ne sont jamais autonomes. Cela veut dire qu'ils requièrent invariablement la présence des segments qui leur servent d'appui, qui les suivent ou qui les précèdent. Les éléments toniques sont capables de former un groupe rythmique. Par contre, ils ne peuvent jamais se substituer à des phrases entières, ce qui les distingue des éléments lexicaux. Les moyens quantificatifs se situent à mi-chemin entre les exposants grammaticaux et lexicaux de la réciprocité. Ils figurent toujours dans la structure d'une phrase à titre d'un groupe rythmique isolé. Par contre, ils ne sont pas capables de remplacer des phrases entières. Ordinairement, leur forme consiste dans la juxtaposition de deux éléments successifs d'une série. Ainsi est formé un contraste qui reflète iconiqument la non-identité de deux participants. L'emploi de ces signes dépasse le cadre de ce qui est considéré dans cet article comme constructions réciproques, ce qui complique sérieusement leur description. Les indicateurs de la réciprocité brièvement décrits ici ne sont pas mutuellement exclusifs dans tous les contextes. Il arrive qu'une seule phrase contienne plusieurs d'entre eux en même temps. La méthode la plus facile de leur analyse consiste à les apparier avec les expressions soumises à la transformation suivant le partage en parties du discours.

\subsection{LA CONVERSION DES CONSTRUCTIONS FONDÉES SUR LES ADJECTIFS}

Moyens quantificatifs. L'emploi de l'un l'autre en compagnie des adjectifs semble dominant du point de vue de sa fréquence. Plus spécifiquement, il s'agit des variantes prépositives de cette expression, les adjectifs français n'étant pas directement combinables avec leurs compléments (exception faite des formes déverbales terminées par -ant, si tant est qu'on y voie des adjectifs, cf. détestant l'un l'autre, respectant l'une l'autre, craignant les uns les autres, etc). L'emploi de ce signe n'est pratiqué qu'à l'intérieur des constructions simples (cf. *Sylvie est jalouse de Pierre l'un de l'autre). Les noms des participants à la relation doivent donc être coordonnés à l'intérieur d'un seul constituant ou bien être comprimés à une seule forme nominale au pluriel. Comme il a déjà été dit, l'emploi du pluriel s'appuie sur l'extraction d'un trait ontologique commun à l'un et à l'autre objet et présuppose une certaine similarité référentielle de leur part. 
Ces deux groupes étaient extrêmement suspicieux l'un envers l'autre.

Admiratifs l'un pour l'autre, les deux peintres conviennent d'un échange de tableaux : deux études des tournesols contre une scène martiniquaise.

Moyens lexicaux. Il s'agit de la combinaison de l'adjectif qui nomme une propriété ou une relation dyadique avec un adverbe ou une locution adverbiale qui véhicule une des significations associables au concept de réciprocité. Cette classe d'expressions renferme entre autres : simultanément, solidairement, réciproquement, mutuellement, analogiquement, tour à tour, en alternance. L'adjectif assume dans ces constructions nécessairement la fonction d'attribut ou d'épithète détachée. Il s'agit donc d'une opération syntaxique, modificative pour le sens. La preuve en est que les adjectifs représentant les reciproca tantum ne tolèrent pas la compagnie des adverbes en question (réciproquement égal, simultanément analogue, également similaire, mutuellement semblable, etc.). Leurs signifiés recèlent déjà le concept d'égalité, de réciprocité, de simultanéité, etc. À l'opposé de la conversion réalisée au moyen de l'un l'autre, les constructions contenant les adverbes réciproques revêtent parfois la forme discontinue. Au niveau syntaxique, l'emploi des signes en question exige que soit introduit dans la phrase le nom d'un nouveau participant à la situation signifiée par l'adjectif. La réciprocité se superpose justement à la relation entre ce participant et celui auquel la référence est faite par le nom accompagnant l'adjectif avant la conversion.

Le comte de Savoie et le dauphin de Viennois étaient mutuellement trop jaloux de leur puissance pour demeurer d'accord.

Sous-estimée par la plupart de nos contemporains, cette montée de l'islam est analogiquement comparable aux débuts du communisme du temps de Lénine.

Tous les peuples sont jaloux au même degré de conserver ce qu'ils possèdent.

Notre peuple est jaloux de conserver ce qu'il possède au même degré que tous les autres peuples.

\subsection{LA CONVERSION DES CONSTRUCTIONS FONDÉES SUR LES NOMS}

Moyens lexicaux. La vocation syntaxique primaire des noms consiste à compléter les positions ouvertes par d'autres termes, c.-à.-d. à se conformer aux propriétés combinatoires de ceux-ci. Ce statut résulte de l'absence, dans la structure des noms, de morphèmes porteurs des indications modales et temporelles. Il existe cependant un emploi secondaire de cette classe de signes où sont cumulés tous les éléments nécessaires d'une prédication complète. L'originalité de ce modèle consiste dans la répartition des informations lexicales, modales et temporelles entre les différents termes. Il est question en ce lieu des collocations verbo-nominales où le rôle du verbe consiste à positionner les noms des participants à la situation dans la structure linéaire de la construction. Les morphèmes flexionnels, obligatoirement attachés au radical verbal, transmettent des indications sur la localisation temporelle de la situation et sur 
l'attitude du sujet parlant envers ce qui est dit. Enfin, les morphèmes grammaticaux faisant partie de la structure du verbe assurent les distinctions aspectuelles. Par contre, c'est l'élément nominal de ces combinaisons qui contribue à préciser la nature de la situation extra-linguistique signifiée par la phrase. Partiellement désémantisé, le verbe n'a qu'un apport secondaire quant à l'essence de toute la relation. Les noms dans ces collocations ne doivent pas être nécessairement réciproques. Leur conversion consiste alors à introduire dans la phrase le troisième élément - un adjectif réciproque (proche, mutuel, égal, analogue, simultané, opposé, rival). Attaché au nom à titre d'épithète, c'est justement cet adjectif qui assure la conversion. Au niveau syntaxique, sa présence dans la phrase entraine la nécessité de nommer un nouveau participant à la situation. L'opération se fait donc en quelques étapes : accusation contre qqn. - porter une accusation contre qqn. - porter des accusations mutuelles. Les constructions simples constituent le cadre syntaxique le plus favorable à ce type de conversion, leurs correspodants discontinus étant moins fréquents.

La victoire aurait pu rester indécise pendant des heures entières, ou la lutte se serait terminée faute de combattants. Bleus et Chouans déployaient une égale valeur.

Les deux commissaires donnent au gouvernement ou au Parlement des avis divergents en ce qui concerne l'incidence de lois sur la protection de la vie privée.

Philippe le Bon et la reine Isabeau de Bavière firent preuve des ambitions rivales.

Moyens quantificatifs. Les collocations verbo-nominales peuvent être soumises à la conversion également grâce à l'emploi de l'un l'autre, toujours avec une préposition. La contribution des éléments constitutifs des combinaisons ainsi formées est la même que dans le cas des phrases contenant des adjectifs. L'emploi de l'un l'autre est aussi limité aux constructions simples. Il existe une contrainte sémantique additionnelle imposée à la conversion réalisée avec ce moyen. Il s'agit des relations qui engagent au moins trois participants et qui font appel au concept de hiérarchie. Celleci est conçue ici comme une sorte de disposition spatiale ou temporelle des éléments (antériorité ou postériorité ; antécédence ou succession). Les relations entre les éléments formant des ensembles ordonnés selon ces principes ne sont pas, à proprement parler, réciproques. Il s'agit plutôt d'une série ou d'une chaîne dont certains éléments jouent un double rôle. P.ex. parmi les quatre individus qui avancent à la queue leu leu, les deux qui sont au milieu se font en même temps précéder par quelqu'un et suivre par quelqu'un d'autre. Par contre, le premier et le dernier de la série ne jouent qu'un seul rôle. Les situations de ce type ne seront pas comptées au nombre de relations réciproques.

L'accueil que les époux se réservent l'un à l'autre est lié à leurs tempéraments violents.

Les deux garçons éprouvent l'un pour l'autre, d'emblée, dans un décor exaltant, un profond dégoût.

Ces petits commerçants ne semblaient ressentir ni crainte, ni jalousie les uns envers les autres, à tel point que chacun pouvait quitter sa boutique et la laisser à la garde de son voisin et concurrent lorsqu'il lui fallait s'éloigner pour un moment. 
Moyens grammaticaux clitiques. Il s'agit de l'emploi de la forme appropriée du pronom datif se. En ce qui concerne les phrases déclaratives, cet élément est chaque fois inséré devant le verbe support. Répandue dans plusieurs langues indo-européennes à titre d'indicateur d'un sens précis de réfléxivité, cette forme a gagné une multitude d'emplois nouveaux. Selon D. Creissels, son statut a évolué vers le rang d'exposant de rôles impartis aux référents des éléments nominaux d'une phrase. L'acquisiton d'un caractère réciproque est due, selon ce chercheur, à l'extension de la notion de réflexivité à une collectivité. Le sens réflexif impliquant toujours une duplicité de rôles (dans Jean se lave, le référent de Jean est, en même temps, l'instigateur de l'action et affecté de celle-ci), il est possible de les échanger avec d'autres individus appartenant au groupe (Creissels, 2006, chapitre 22). C'est ainsi qu'est née la valeur réciproque de $s e$. L'emploi de cette forme n'écarte pas toutes les autres interprétations des constructions pronominales. En théorie, les collocations verbo-nominales avec un sujet au pluriel ou dont les membres sont coordonnés à l'intérieur d'un seul constituant, peuvent avoir une lecture réflexive (Jacqueline et Sylvie se donnent beaucoup de conseils / se donnent des coups d'horions ; voir Borillo, 1971, p. 26). C'est pour cette raison qu'on introduit dans les phrases construites conformément à ce modèle un adjectif épithète du type mutuel, réciproque accompagnant le nom de relation.

Des deux côtés du continent, les deux religions se jettent un défi d'intolérance. Catholiques et protestants sont opprimés là où ils ne peuvent être oppresseurs.

Les hommes des cavernes s'assénaient des coups de bâton pour une femme ou pour la dernière aile de ptérodactyle.

Ils ne vivent pas ensemble mais se consacrent beaucoup de temps. Elle lui fait changer ses habitudes alimentaires. Plus de viandes rouges.

\subsection{LA CONVERSION DES CONSTRUCTIONS FONDÉES SUR LES VERBES}

Moyens grammaticaux (clitiques et toniques). Tous les éléments nécessaires à une prédication complète faisant partie de la structure d'un verbe, les transformations des phrases bâties sur cette classe de signes sont les moins compliquées. Une première variante de conversion consiste dans l'emploi en compagnie d'un verbe de la forme appropriée du pronom clitique se. Cette transformation, appliquée en français uniquement dans des constructions simples, produit des phrases potentiellement ambigues, susceptibles de recevoir tantôt une lecture réflexive, tantôt réciproque. C'est pour cette raison que la conversion des verbes ne se fait pas uniquement au moyen de cet élément. Il se crée de la sorte des configurations de plusieurs termes, conformément au mécanisme de co-signification décrit ci-dessus. Cette ambiguité peut être levée soit grâce à l'emploi d'un adverbe réciproque (Ils se détestaient mutuellement), soit grâce à l'emploi d'un pronom tonique avec la préposition entre (Ils se détestaient entre eux). Ces deux éléments ont également un autre rôle à jouer. Sauf le rejet d'une lecture réflexive, ils affirment l'existence d'une relation entre les objets nommés dans 
la phrase et non entre un référent collectif et un autre participant, non exprimé ouvertement. Employé de cette façon, l'élément entre eux, entre nous, etc. est susceptible d'accompagner mêmes les verbes de la classe reciproca tantum (Les deux garçons rivalisaient entre eux d'arrache-pied; Les deux garçons rivalisaient [avec quelqu'un d'autre]).

Ils se sont abondamment congratulés sur la naissance de leurs fils.

Partout où les avant-postes russes et autrichiens se rencontraient, ils se tendaient la main en se promettant de servir bientôt ensemble.

Les officiers sont couchés et lisent ou se plaignent entre eux de leur inactivité. Tous lèvent la tête quand un coup de téléphone résonne.

Dans ce lieu paradisiaque, les couples s'embrassent et se promettent monts et merveilles avant le moment fatidique de la séparation.

Moyens lexicaux. Alors qu'un des moyens de convertir les noms consiste à les faire accompagner d'un adjectif épithète porteur d'un des concepts réciproques, les verbes acquièrent cette nouvelle valeur grâce à l'emploi des adverbes. Leur répertoire ne diffère pas de celui qui servait à la transformation des phrases bâties sur les adjectifs-attributs. Il est une nouvelle fois question ici de parallèlement, réciproquement, mutuellement, analogiquement, en alternance, concurremment. Appartiennent à cet ensemble des locutions adverbiales dont une partie importante suit le modèle : " nom d'une partie du corps - à - nom d'une partie du corps ». P.ex.: nez à nez, coude à coude, dos à dos, face à face. Il doit être remarqué que les adverbes déadjectivaux et les adjectifs réciproques ne s'opposent pas par leur sens. Par contre, c'est le contexte, conçu comme la classe d'expressions avec lesquelles les adverbes et les adjectifs sont combinables, qui les distingue. Cette situation porte en linguistique le nom de distribution complémentaire.

D'habitude les champions se tenaient face à face et s'assenaient des coups terribles sans finesse aucune, jusqu'à ce que l'un des deux, épuisé, baisse sa garde et encaisse enfin sur le crâne le coup fatal qui mettait fin au combat.

Les soldats et les prisonniers marchaient, ils étaient côte à côte.

Les deux prétendants à l'investiture du Parti démocrate pour l'élection restent au coude à coude et ont chacun des raisons de se réjouir des résultats du «super-mardi ».

Nous savons, d'ailleurs, pour quelles raisons ces deux phénomènes se développent parallèlement.

[...] la sphère de l'action sociale irait donc de plus en plus en se rétrécissant, car elle n'aurait plus d'autre objet que d'empêcher les individus [...] de se nuire réciproquement.

Moyens quantificatifs. L'un l'autre s'avère le moyen le plus universel, capable de convertir toutes les classes d'expressions susceptibles de nommer des situations dyadiques non-réciproques. À l'opposé des phrases bâties sur les noms et les adjectifs, cette expression ne doit pas contenir une préposition en compagnie des verbes. Comme précédemment, les verbes soumis à la transformation ne peuvent pas signifier des relations séquentielles appliquées à au moins trois individus. L'un l'autre qu'on voit 
apparaître même avec les reciproca tantum en vue de signaler que la relation concerne les objets nommés dans la phrase, n'apparaît que dans les constructions simples.

Mais toujours il entraînait cramponnés à ses flancs les deux hommes qui luttaient pour se tuer l'un l'autre.

Dieu et la nature agiraient sans raison en traitant l'un autrement que l'autre.

Ce que nous étions l'un pour l'autre, nous le sommes toujours.

Moyens grammaticaux (affixaux). Puisque le présent article est consacré surtout aux variantes syntaxiques, il reste peu de choses à dire sur l'affixation réciproque en français. Le descendant du co- (préfixe) et du cum (préposition) latins figure dans la structure d'à peu près trois cents verbes français. Pourtant, ils sont souvent entièrement ou partiellement lexicalisés, ce qui veut dire que pour un usager ne disposant pas d'une connaissance approfondie de l'histoire de sa langue, le sens de ces verbes n'est plus senti comme compositionnel. S'y ajoutent des formations à sens collectif qu'il ne faut pas confondre avec les verbes réciproques (concélébrer, cohériter, etc.). Par contre, les préfixes entre- et inter- ont une productivité plus large. Les verbes qu'ils modifient s'accompagnent invariablement de l'élément se et ne fondent que des constructions simples.

Il y a beaucoup de romanciers français qui s'entradmirent et se dédient des articles de presse élogieux.

Poissons, roseaux, bois, transport fluvial et tous les organismes vivants sont dès lors en interdépendance.

Il convient de souligner que l'état de choses actuel diffère sensiblement de ce qui s'observe en ancien français. Premièrement, dans les textes médiévaux l'élément se n'est pas toujours présent en compagnie des noms de relations réciproques dérivées. Deuxièmement, les collocations verbo-nominales exhibent des différences de structure sérieuses par rapport à l'état actuel. En témoigne le fragment ci-dessous.

Uns vavasors andemantiers antra leanz,

qui mout lor nut,

qui monseignor Gauvain conut,

si les trova antrebeisant

et mout grant joie antrefeisant (Perceval, 5766-5770)

\section{CONCLUSIONS}

En dépit d'une profusion récente de publications consacrées à la réciprocité, le problème y est généralement réduit à l'étude des verbes. Cette pratique rétrécit le panorama de ce domaine de recherches linguistiques. D'autres classes morphologiques contribuent également à l'expression de ce sens. Néanmoins, cette tendance des chercheurs à ne s'intéresser qu'aux verbes semble partiellement justifiée. L'emploi prédicatif des noms et des adjectifs est secondaire, en ce qu'il est toujours tributaire 
des configurations, parfois très complexes, de plusieurs autres termes. Les collocations verbo-nominales, sans être exceptionnelles, érigent les noms au rang de prédicats. Il en est de même de l'emploi des adjectifs en guise d'attributs. Pourtant, une description adéquate de la réciprocité demande que soient prises en compte toutes les variantes, mêmes celles qui sont syntaxiquement compliquées.

L'autre remarque concerne la disproportion entre la richesse des moyens syntaxiques qui assurent le passage vers la réciprocité et la pauvreté des outils morphologiques. Alors que les pronoms clitiques apparaissent assez régulièrement dans des constructions réciproques dérivées, l'emploi des éléments toniques et affixaux reste extrêmement limité. L'ambiguïté qui caractérise systématiquement les constructions simples contenant la forme se entraîne la nécessité de recourir à d'autres moyens, le plus souvent lexicaux. Sont formées de la sorte des configurations de plusieurs expressions. Leur étude appartient au champ d'investigation de la syntaxe.

\section{BIBLIOGRAPHIE}

Borillo, A. (1971). Remarques sur les verbes symétriques français. Langue française, 11, 17-31.

Chrétien de Troyes (s.d.). Le Conte du Graal (Perceval). Récupéré sur Université d'Ottawa Faculté des Arts - Laboratoire de français ancien: http://www.uottawa.ca/academic/arts/lfa/activites/ textes/perceval/galv.htm

Creissels, D. (2006). Syntaxe générale - une introduction typologique. II - La phrase. Paris : Hermès Science-Lavoisier.

Pogonowski, J. (1993). Combinatory Semantics. Poznań: Wydawnictwo Naukowe UAM.

Wierzbicka, A. (1993). La quête des primitifs sémantiques : 1965-1992. Langue Française, 98, 9-23.

Zaliznjak, A.A., \& Shmelev, A.D. (2007). Sociativity, conjoining, reciprocity and the Latin prefix com-. Dans V.P. Nedjalkov, Reciprocal Constructions (Typological Studies in Language 71) with the assistance of Emma Š. Geniušiene and Zlatka Guentchéva (vol. 1, pp. 209-229). Amsterdam - Philadelphia: John Benjamins Publishing Company. 